\title{
No interference of the $1,3-\beta$-D-glucan containing nutritional supplement ImunixX with the 1,3- $\beta$-D-glucan serum test
}

\author{
Isabel Spriet, ${ }^{1}$ Stephanie Desmet, ${ }^{2}$ Ludo Willems ${ }^{1}$ and Katrien Lagrou ${ }^{2}$ \\ ${ }^{1}$ Pharmacy Dpt., University Hospitals Leuven, Leuven, Belgium and ²Department of Medical Diagnostic Sciences, University Hospitals Leuven, Leuven, Belgium
}

\section{Summary}

The detection of 1,3- $\beta$-D-glucan serum levels may permit establishing the diagnosis of invasive fungal infections more early. We tested in six healthy volunteers whether the intake of a $1,3-\beta$-D-glucan-containing nutritional supplement leads to false-positive $1,3-\beta$-D-glucan levels. All levels were negative, even in two different dosing regimens.

Key words: Antigen test, identification, cell wall.

\section{Background}

Early diagnosis of invasive fungal infection (IFI) in neutropenic subjects has the potential to increase antifungal therapeutic response. Histopathological demonstration of organisms in tissue specimens or growth of fungal agents in culture media is still the gold standard method, but obtaining such specimens may be difficult. ${ }^{1,2}$

$1,3-\beta$-D-glucan is part of the outer cell wall of pathogenic fungi with the exception of Zygomycetes and Cryptococcus species. Viruses, as well as human cells, do not contain 1,3- $\beta$-D-glucan. Glucans are released from fungal and bacterial cell walls into the systemic circulation of patients with (invasive) infection. It has been shown that $1,3-\beta$-D-glucan determination, when combined with risk stratification and imaging, is useful in permitting the early diagnosis of IFI. ${ }^{1}$

Recently, a nutritional supplement ImunixX (ixX Pharma) is promoted as dietary supplement to stimulate and restore the immune system. ${ }^{3}$ This supplement will be targeted by the company to, amongst other populations, haematological/oncological patients [personal communication IS - ixX pharma]. The active substance in ImunixX is 1,3-1,6- $\beta$-D-glucan (derived from the cell wall of Pleurotis ostreatus). ${ }^{4}$

Detection of 1,3- $\beta$-D-glucan serum levels has been shown to be a faster method than culture in the

Correspondence: I. Spriet, PharmD, Pharmacy Department, University Hospital Leuven, Herestraat 49, 3000 Leuven, Belgium.

Tel.: +32 1634 1261. Fax: +321634 3085 .

E-mail: isabel.spriet@uzleuven.be

Accepted for publication 21 April 2010 diagnosis of IFI, but as for all immunological tests, some non-specific cross-reactions have been reported. Falsepositive results have been shown, amongst other factors, in patients with bacterial infections, in patients treated with immunological preparations such as albumins or globulins or when cellulose membranes or filters are used (i.e. during haemodialysis). In accordance to false positivity reported with the galactomannan test, false positivity could also expected in relation to diet or the intake of dietary supplements. ${ }^{1}$ We examined if the daily oral intake of the ImunixX dietary supplement could lead to false-positive 1,3- $\beta$-D-glucan levels leading to interference with the diagnosis of IFI.

\section{Methods}

The study was performed during a 7-day period in six healthy volunteers (two male subjects, four female subjects; mean age: 30 years). The volunteers were randomly divided into two groups to test two different dosing regimens, as recommended by the company, one for treatment (high dose) and one for prophylaxis (low dose). ${ }^{3}$ Three volunteers were taking the high-dose regimen, i.e. $500 \mathrm{mg}$ of 1,3-1,6- $\beta$-D-glucan daily during 5 days followed by $100 \mathrm{mg}$ daily during 2 days. The second group of three volunteers took $100 \mathrm{mg}$ daily during 7 days.

Three blood samples were taken from each volunteer. The first sample was taken before the administration of ImunixX (day 0), and the second and third samples were taken, respectively, on days 5 and $7,1 \mathrm{~h}$ after ingestion. Samples were centrifuged and serum was frozen at $-20{ }^{\circ} \mathrm{C}$ until analysis. 
The dietary supplement was ingested on an empty stomach to avoid interaction with food. During the study period, mushrooms were avoided to exclude false positivity due to diet.

$1,3-\beta$-D-glucan levels were determined as previously published using the Fungitell 1,3- $\beta$-D-glucan detection assay (Associates of Cape Cod), which has been shown to be a highly sensitive and specific diagnostic tool for IFI, when a cut-off of serum 1,3- $\beta$-D-glucan level of 60 or $80 \mathrm{pg} \mathrm{ml}^{-1}$ is chosen. ${ }^{5,6}$ The Fungitell assay reacts equally with both 1,3- $\beta$-D-glucan and 1,3-1,6- $\beta$-Dglucan. ${ }^{5}$

The study was approved by the hospital's Ethics Committee, and all participants signed the informed consent.

\section{Results and Discussion}

$1,3-\beta$-D-glucan serum levels were negative in all volunteers during the period of intake of the ImmunixX supplement, both in the high-dose and prophylacticdose regimens. In the low-dose group, mean levels [and ranges] were 35.6 [6-84], 31.3 [21-50] and 20.3 [1138] $\mathrm{pg} \mathrm{ml}^{-1}$, on days 0,5 and 7 respectively. In the high-dose regimen, mean levels [and ranges] were 26.5 [11-42], 16.3 [11-21] and 30.3 [10-45] $\mathrm{pg} \mathrm{ml}^{-1}$ on days 0,5 and 7 respectively. Only in subject no. 6 , the day 0 sample was $84 \mathrm{pg} \mathrm{ml}^{-1}$. This elevated $\beta$-D-glucan result cannot be easily clarified, maybe it was related to the intake of other fungi-containing food, as only mushrooms were restricted. However, a positive serum level can also reflect an aspecific reaction. The specificity of the Fungitell assay has been shown to be $92.5 \%$ in 40 healthy blood donors. ${ }^{7}$ It was certainly not related to the intake of the ImunixX, as the sample was taken before ingestion of the supplement.

During the last 20 years, several mushroom extracts, containing amongst other substances $\beta$-D-glucans, were promoted for their potential anticancer effects, such as induction of apoptosis or modulation of the immune response. ${ }^{8,9}$ Ruling out cross-reactivity with the $1,3-\beta$ D-glucan serological test is important, especially in this setting where 1,3- $\beta$-D-glucan is used as a biomarker for earlier diagnosis of IFI.

According to the product information of this specific supplement, ImunixX stimulates the host immune response by binding the M-cells (microfold cells) found in the epithelium of the Peyer's patches in the duodenum. These M-cells are capable of transporting 1,3-1,6$\beta$-D-glucan across the epithelial barrier from the gut lumen to the macrophages, leading to a stimulated mucosal immunity. ${ }^{3}$
It seems from our results that ImunixX can probably be safely used in patients with an intact mucosal barrier. However, this should be interpreted with care, as this was tested in only six volunteers, obviously the main limitation of our study. Moreover, it remains unclear whether these results can be extrapolated to patients with mucositis or graft-vs.-host-disease, as the absorption of 1,3-1,6- $\beta$-D-glucan may be enhanced because of increased gastrointestinal permeability.

\section{Conflict of Interest}

The ImunixX tablets were kindly provided by ixXpharma. No conflicting interests to declare.

\section{References}

1 Kedzierska A, Kochan P, Pietrzyk A, Kedzierska J. Current status of fungal cell wall components in the immunodiagnositics of invasive fungal infections in humans: galactomannan, mannan and 1,3-D-glucan antigens. Eur J Clin Microbiol Infect Dis 2007; 26: 755-66.

2 Year H, Sendid B, Francois N, Camus D. Contribution of serological tests and blood culture to the early diagnosis of systemic candidiasis. Eur J Clin Microbiol Infect Dis 2001; 20: $864-70$.

3 Bauerova K, Paulovicova E, Mihalova D, Svik K, Ponist S. Study of new ways of supplementary and combinatory therapy of rheumatoid arthritis with immunomodulators. Glucomannan and Imunoglucan in adjuvant arthritis. Toxicol Ind Health 2009; 25: 329-35.

4 Product Information Imunixx. URL http://www.ixx.be [accessed on 26 October 2009].

5 Odabasi Z, Mattiuzzi G, Estey E et al. $\beta$-D-glucan as a diagnostic adjunct for invasive fungal infections: validation, cutoff development, and performance in patients with acute myelogenous leukemia and myelodysplatic syndrome. Clin Infect Dis 2004; 39: 199-205.

6 Ostrosky-Zeichner L, Alexander BD, Kett DH et al. Multicenter clinical evaluation of 1,3- $\beta$-D-glucan assay as an aid to diagnosis of fungal infections in humans. Clin Infect Dis 2005; 41: 654-9.

7 Persat F, Ranque S, Derouin F, Michel-Nguyen A, Picot S, Sulahian A. Contribution of the (1-3)- $\beta$-D-Glucan assay for diagnosis of invasive fungal infections. J Clin Microbiol 2008; 46: 1009-13.

8 Shomori K, Yamamoto M, Arifuku I, Teramachi K, Ito H. Antitumor effects of a water-soluble extract from Maitake (Grifola frondosa) on human gastric cancer cell lines. Oncol Rep 2009; 22: 615-20.

9 Harhaji Trajkovic LM, Mijatovic SA, Maksimovic-Ivanic DD et al. Anticancer properties of Ganoderma lucidum methanol extracts in vitro and in vivo. Nutr Cancer 2009; 61: 696-707. 